Research Article

\title{
The Effect of Plasmonic Nanostructures Prepared by Electrical Exploding Wire Technique on the Optical Properties of R6G Dye
}

\author{
Fouad Hamzah ${ }^{-}$Hammad Humud \\ Department of Physics, College of Science, University of Baghdad, Baghdad, Iraq. \\ Corresponding authors. E-mail: fghhamzah@gmail.comTel.: +9647717170443, +9647808511402
}

Received: Jun. 8, 2020; Accepted: Oct. 19, 2020; Published: Apr. 23, 202I

Citation: Fouad Hamzah, Hammad Humud,The Effect of Plasmonic Nanostructures Prepared by Electrical Exploding Wire Technique on the Optical Properties of R6G Dye. Nano Biomed. Eng., 202I, I3(2): I37- 149.

DOI: 10.5101/nbe.v13i2.p137-149.

\begin{abstract}
One of the rapidly growing fields of nanotechnology is its manipulation of laser dyes' properties using nanoparticles and nanostructures due to its various applications, ranging from biomedical imaging to green energy. Silver nanoparticles (Ag NPs) of various concentrations and nanostructures with silver nanowire (Ag NW) were prepared using an electrical exploding wire technique (EEW) and was mixed with a fixed concentration of R6G dye. The behavior of energy transfer from the dye molecules (R6G) to nanomaterials (Ag NPs or plasmonic nanostructures) was examined using fluorescence spectra. The experimental results showed that the fluorescence intensity quenched with increasing concentration and density number of Ag NPs. The distance between the dye molecules and the nanostructures was studied, which was found to decrease as the concentration and density number of Ag NPs increased in the mixture. The energy transfer efficiency of nanostructures was compared. It was obtained that nanostructure (Ag NW@PDA@Ag NPs) achieved the best energy transfer efficiency of 85\%. Our results indicated that this nanostructure could sense a distance around the metal nanoparticles $(\approx 27.2$ $\mathrm{nm}$ ); thus the nanoparticle-based surface energy transfer (NSET) mechanism is dominated rather than Förster resonance energy transfer (FRET) mechanism. This process is affected by concentration increasing of Ag NPs and coated morphology of Ag NWs by polydopamine (PDA) layer decorated by Ag NPs. The findings can be utilized in the large field of bio diagnostics and biochemistry. Regardless of bio-applications, the quenching mechanisms and rates are also of interest for SERS, (dye-sensitized) solar cells or nanooptics. However, we see the best potential in bio-sensing by managing the quenching rate by adjusting the shape or the concentration of nanostructures.
\end{abstract}

Keywords: Fluorescence quenching, Plasmonic nanostructures, Nanoparticles, Rhodamine 6G (R6G) dye, Density number of Ag NPs, Energy transfer efficiency

\section{Introduction}

Nanoparticles can be synthesized using electroexploding wire (EEW) technique, while surrounded by a non-flammable liquid medium such as deionized water. The particles evaporated during the explosion will condense into the liquid more efficiently than surrounding air. Properties of nanoparticles created by EEW rely upon numerous parameters, which include wires' material and diameter, features of the electrical circuit and the surrounding medium [1, 2].

The fluorescence characteristics of organic dye molecules are highly impacted if these molecules are placed on the surface of noble metallic nanoparticles, 
such as silver and gold nanoparticles (Ag NPs and Au NPs) [3]. So, the effect of metallic nanoparticles on organic dye molecules has taken a wide field of research in the field of materials science, random laser, imaging and mapping applications and chemical or biomedical applications [4-7]. These applications are most likely a direct result of the unique surface properties of Ag NPs including surface-enabled molecular interactions and chemical reactions $[8,9]$. Such interactions can result in biological consequences such as the detection of various toxins $[10,11]$, and antimicrobial activities [12, 13]. Two widely studied surface phenomena of Ag NPs are Surface Enhanced Raman Scattering (SERS) [14, 15], and protein corona formation [16, 17]. Therefore, it is very important to understand the surface interactions of Ag NPs with small organic molecules $[18,19]$. By enhancement of fluorescence spectra, better sensitivity and a high signal to noise ratio can be obtained in the molecular sensing/imaging, while quenching of the fluorescence spectra can be used for negative sensing [20-22]. There are three essential mechanisms that determine the fluorescence characteristics in the presence of nanoparticles: electron transfer, Förster resonance energy transfer (FRET) and nanoparticles-based surface energy transfer (NSET). All of them have been widely studied and they are all dependent upon the distance. The effective distance in FRET mechanism is mainly limited to a maximum of $10 \mathrm{~nm}$ [23-25]. NSET mechanism dominates at average distances up to about $30 \mathrm{~nm}$ [26]. The electron transfer mechanism takes place only when the distance between the NP surface and the dye molecules is shorter than $1.5 \mathrm{~nm}$ [27]. Generally, in the mixtures of dye, the acceptor and the donor are deemed as point dipoles, and the resonance energy transfer between them is clarified in terms of the Forster Resonance Energy Transfer (FRET) mechanism. However, some of the latest studies indicates that the Nanoparticles Surfaces Energy Transfer (NSET) is more convenient to clarify the transfer of energy between the dye molecule and metallic nanoparticle [23, 28, 29]. Both these mechanisms can serve as a spectroscopic ruler about the nanoparticles and can take a look at the surrounding or the dielectric environment about the nanomaterial in a dye-nanomaterial mixture. The FRET mechanism in dye-NPs systems operates at a distance of less than $10 \mathrm{~nm}$ (Forster's radius, $\mathrm{R} 0 \approx 6$ $\mathrm{nm})$, while the NSET mechanism can sense a higher distance about the metallic NPs ( $22 \mathrm{~nm})$. In NSET mechanism, the nanoparticle-fluorophore interaction is considered as the interaction between a dipole and a nanoparticle surface compared with the dipole-dipole interaction in FRET. The influence of interaction depends on different factors such as the shape and size of the nanoparticles, the relative orientation of dipole moments and overlap of the plasmon band of the nanoparticles with the emission and absorption bands of the dye [30-32].

The studies implemented using dye-Ag NPs mixture showed quenching as well as the enhancement of intrinsic photoluminescence (PL) of the dye molecules, depending on the type of test and the distance between the NPs and the dye molecules [33, 34]. As reported in previous study at shorter distances, the fluorescence quenching dominates over enhancement due to the mechanism of the efficient nonradiative energy transfer among the dye molecules and the metallic nanoparticles [35].

The novelty of this work is that have been fabricated nanostructures in a fast, simple, lowcost and environmentally friendly technique without chemical additives. As well as know the mechanisms adopted for energy transfer among dye molecules and nanoparticles or nanostructures prepared with EEW technique. Likewise, knowing the AgNW@PDA@ AgNPs nanostructures that achieve the best energy transfer efficiency, for later use as biological and chemical sensors and for food security applications [36].

\section{Experimental}

\section{Materials and characterization instruments}

The Rhodamine 6G (R6G) $\left(\mathrm{C}_{28} \mathrm{H}_{31} \mathrm{~N}_{2} \mathrm{O}_{3} \mathrm{Cl}\right.$, molecular weight $479.02 \mathrm{~g} / \mathrm{Mol}$ ) was purchased from EXCITON CHEMICAL CO. INC. The silver bullion was purchased from the Central Bank of Iraq by a jeweller. Ag NWs were purchased from XFNANO with an average diameter of $90 \mathrm{~nm}$ and length of 1micron (500 mg dissolved in $25 \mathrm{~mL}$ water), where the concentration was $\approx 0.2 \mathrm{M}$. Dopamine hydrochloride, tris-base $(\geq 99.9 \%)$ was purchased from SigmaAldrich. The R6G dye solution was prepared by gravimetric method, where the prepared concentrations of R6G dye were from $1 \times 10^{-1}$ to $1 \times 10^{-6} \mathrm{M}$. From silver bullion, a wire of $0.3 \mathrm{~mm}$ diameter and the plate of $3 \mathrm{~cm} \times 2 \mathrm{~cm} \times 3 \mathrm{~mm}$ dimensions were used as electrodes in an electrical exploding wire technique. The details of internal nanostructures features were 
examined by X-ray diffraction (XRD), where a few drops from aqueous colloid of Ag NPs, Ag NWs, AgNw@AgNPs,AgNW@PDA and AgNW@PDA@ AgNPs nanostructures were dried on glass substrate, and X-ray diffraction (XRD) data were taken in the range of 10-90 $2 \theta$ degree. The XRD pattern was performed with XRD 6000 Shimadzu. X-ray diffractometer using a Radiation: $\mathrm{CuK} \alpha_{1} \lambda: 1.5406 \AA$. The morphologies of the Ag NWs and AgNW@AgNPs nanostructure were characterized with a field-emission scanning electron microscope (FE-SEM). Analysis was performed on samples deposited onto a rinsed silicon wafer and dried under ambient conditions. The particle size and morphologies of Ag NPs, AgNW@ PDA, and AgNW@PDA@AgNPs nanostructures were further characterized by scanning transmission electron microscopy (STEM) and performed using FESEM, STEM Nova NanoSEM 450 with accelerating voltage of $1000 \mathrm{kV}$. The concentration of prepared Ag NPs by EEW technique was measured using AA-7000 Atomic Absorption Spectrophotometer Shimadzu. The absorption spectra of the prepared nanostructures at a different concentration before and after mixing them with the R6G were measured in the wavelength range of $300-800 \mathrm{~nm}$ at room temperature for all samples using Shimadzu-1800 UVVis double beam spectrophotometer. Fluorescence spectra of the prepared samples were recorded using Spectro-fluorophotometer (Shimadzu RF-5301 PC). Fluorescence spectra of the R6G dye were measured before and after mixing it with varied concentrations of the plasmonic nanostructures.

\section{Preparation of Ag NPs by EEW technique}

The role and mechanism of EEW to generate Ag NPs: $30 \mathrm{~mL}$ of deionized water was placed in a shock wave resistant container with a magnetic stirring for all time of preparation at room temperature as shown in Fig. 1. Preparation of Ag NPs was achieved by exploding a high purity Ag wire (0.3 mm in diameter) against $\mathrm{Ag}$ plate that was held in $82.2 \mathrm{~V}$ respect to the wire and 100 A steady current to getting the Ag NPs. The electric exploding of wire was done when the wire is connected to the plate mechanically. So, the weight of the Ag NPs prepared is $30 \mathrm{~mL}$ at a concentration of 2 $\times 10^{-4} \mathrm{M}$. It was diluted to obtain other concentrations.

\section{Preparation of AgNW@AgNPs nanostructures}

The role and mechanism of EEW to generate Ag NPs, and simultaneously decorate them on the surface of Ag NWs: To prepare the Ag NWs decorated by Ag NPs, $1.25 \mathrm{~mL}$ of aqueous colloid of the Ag NWs (0.2 M) was dispersed by $30 \mathrm{~mL}$ of deionized water. Magnetic stir has been used of aqueous colloid of the Ag NWs for $30 \mathrm{~min}$ at room temperature, and then ultrasonicated for 15 min for obtaining a homogeneous aqueous colloid and ensuring complete dispersion. The final aqueous colloid of Ag NWs were placed in resistant container of the shock wave with a magnetic stirring for all time of preparation at room temperature as shown in Fig. 1. Preparation of Ag NPs decorated Ag NWs was achieved by exploding a high purity Ag wire (0.3 $\mathrm{mm}$ in diameter) against Ag plate that was held in $82.2 \mathrm{~V}$ respect to the $\mathrm{Ag}$ wire and $100 \mathrm{~A}$ steady current to getting the AgNW@AgNPs nanostructures (e.g. Metal-metal nanostructures). When the explosion occurs, the Ag NPs will be generated and move at a high speed (hypersonic) forming a shock wave within the surrounding colloid (Ag NWs). These highspeed moving nanoparticles will collide with the surrounding colloid and stick tightly to its surface to form plasmonic nanostructures AgNW@AgNPs. As a result of the explosion of the silver wire inside the surrounding colloid, Ag NWs were decorated with Ag NPs to form metal-metal nanostructures AgNW@ AgNPs. In this method, the number of Ag NPs that

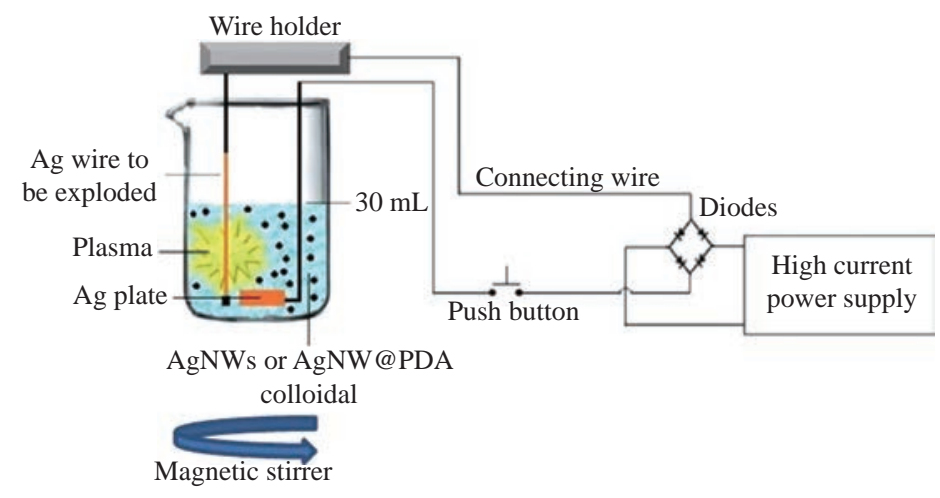

Fig. 1 Experimental set-up for fabricating Ag NPs, AgNW@AgNPs and AgNW@PDA@AgNPs nanostructures. 
has been decorated on the surface of Ag NWs was dependent on the number of explosions, and also the distribution of them on the surface of Ag NWs was random. The @ symbol represents the adhesion of Ag NPs to the surface of Ag NWs. A number of explosions $(5,10,15$ and 20$)$ were generated to get three different samples of the plasmonic nanostru`ctures AgNW@ AgNPs have different number of Ag NPs on the surface. The resulting nanostructures were collected by centrifugation for separating the fabricated plasmonic nanostructures AgNW@AgNPs from Ag NWs and free Ag NPs that did not decorate on the surface of Ag NWs.

\section{Preparation of the AgNW@PDA@AgNPs nanostructures}

The role and mechanism of EEW to generate Ag NPs, and simultaneously decorate them on the surface of silver nanowires coated with polydopamine layer (AgNW@PDA): In this part of the preparation there were two parts of the work (firstly coating Ag NWs and secondly decorating it after coating). In coating Ag NWs, $1.25 \mathrm{~mL}$ of aqueous colloid of the Ag NWs $(0.2 \mathrm{M})$ was dispersed in $30 \mathrm{~mL}$ of deionized water with magnetic stirring for $30 \mathrm{~min}$ at room temperature and then ultra-sonicated for 15 min for the purpose of ensuring complete dispersion. Then $0.05 \mathrm{~g}$ of dopamine hydrochloride powder were added into the aqueous colloid of the Ag NWs. The PH of the final reaction solution was adjusted to 8.5 by adding Tris$\mathrm{HCl}$ buffer solution with a gently stirring speed at room temperature for $24 \mathrm{~h}$. After the color of aqueous colloid is starting to become brown, that mean the polydopamine has been coated the Ag NWs. After centrifuging of solution at $4000 \mathrm{rpm}$ and washing with deionized water several times, the AgNWs@PDA were obtained and stored in deionized water to be used at another time.

In decorating AgNWs@PDA, the PDA-coated Ag NWs were dispersed in $30 \mathrm{~mL}$ deionized water with ultrasonicating. The final aqueous colloid of the AgNWs@PDA was placed in resistant container of the shock wave with a magnetic stirring for all time of preparation at room temperature as shown in Fig. 1. Preparation Ag NPs decorated AgNWs@PDA was achieved by exploding a high purity Ag wire $(0.3$ $\mathrm{mm}$ in diameter) against Ag plate that was held in $82.2 \mathrm{~V}$ respect to the wire and $100 \mathrm{~A}$ steady current to getting the AgNW@PDA@AgNPs nanostructures (e.g. Metal-insulator-metal). When the explosion occurs, the Ag NPs will be generated and move at a high speed (hypersonic) forming a shock wave within the surrounding colloid (AgNWs@PDA). These high-speed moving nanoparticles will collide with the surrounding colloid and stick tightly to its surface to form plasmonic nanostructures AgNW@ PDA@AgNPs. As a result of the explosion of the silver wire inside the surrounding colloid, AgNw@ PDA nanostructures are decorated with Ag NPs to form metal-insulator-metal nanostructures AgNW@ PDA@AgNPs. In this method, the number of Ag NPs (density number of Ag NPs) that have been decorated on the surface of AgNWs@PDA is dependent on the number of explosions, and also the distribution of them on the surface of AgNWs@PDA was random. A different number of explosions (5, 10, 15 and 20) were generated to get three different samples of the plasmonic nanostructures AgNW@PDA@AgNPs have different density number of Ag NPs on the surface. The resulting nanostructures were collected by centrifugation for separating the fabricated plasmonic nanostructuresAgNW@PDA@AgNPs fromAgNWs@ PDA and free Ag NPs that did not decorate on the surface of AgNWs@PDA.

\section{Preparation of samples for fluorescence test}

Fluorescence test was performed using $1 \mathrm{~cm}$ quartz cuvette transparent from four sides. The fluorescence test in this research was in two parts (increasing the concentration of Ag NPs and Ag NWs then increasing the density number of Ag NPs on surface of Ag NWs or PDA layer). In the first part, increasing the concentration of the each of the Ag NPs and Ag NWs was tested and its effect on the fluorescence properties of the R6G dye. While in the second part, the effect of increasing the density number of Ag NPs on the surface of prepared nanostructures was tested on the fluorescence properties of R6G dye (increasing the number of 5, 10, 15 and 20 explosions that leading to increasing in density number of Ag NPs on the surface of nanostructures AgNW@AgNPs and AgNW@ PDA@AgNPs). The excitation wavelength was set at $532 \mathrm{~nm}$ with a split width of $2 \mathrm{~nm}$. The mixing ratio was $2: 1$, where $2 \mathrm{~mL}$ of R6G dye was mixed with 1 mL from each of Ag NPs, Ag NWs, AgNW@AgNPs and AgNW@PDA@AgNPs.

\section{Results and Discussion $X$-ray diffraction patterns of samples}

Fig. 2 shows typical XRD patterns of Ag NPs, AgNWs,AgNW@AgNPs,AgNW@PDA and 


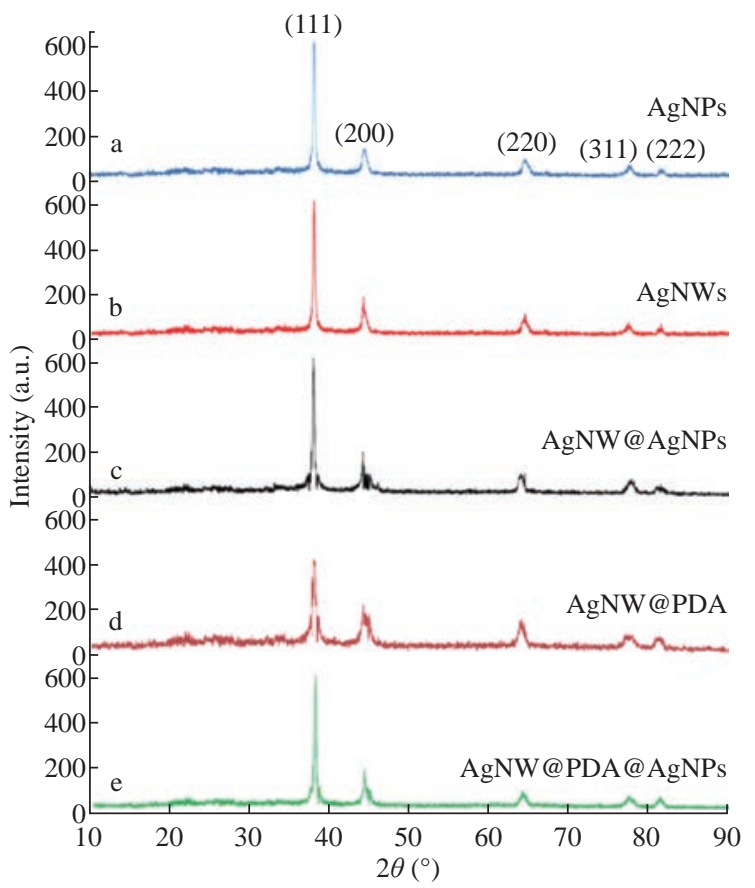

Fig. 2 X-ray diffraction patterns of (a) Ag NPs, (b) Ag NWs, (c)AgNW@AgNPs, (d)AgNW@PDA and (e)AgNW@PDA@ AgNPs nanostructures.

AgNW@PDA@AgNPs nanostructures. The results demonstrated that the prepared Ag NPs,AgNW@ AgNPs and AgNWs@PDA@AgNPs nanostructures are having face-centred cubic phase for Ag metal. All patterns have 5 peaks with a slight difference in the peak location of each sample, were observed at $2 \theta$ degrees of $38.116^{\circ}, 44.227^{\circ}, 64.425^{\circ}, 77.472^{\circ}$ and $81.536^{\circ}$ and have been indexed to hkl values of (111), (200), (220), (311) and (222), respectively, indicating that the prepared Ag NPs, AgNW@AgNPs and AgNW@PDA@AgNPs nanostructures by EEW technique no oxidation had occurred during and after EEW and preserved the high purity and good crystalline nature that was identical to JCPDS card No. 4-0783. The peak position of the XRD pattern is similar to that for standard bulk silver.

\section{EDX element analysis test}

Fig. 3 illustrates the EDX element analysis of Ag NW before and after its coating with PDA layer and after it has been exposed to 20 explosions. EDX analyses showed strong peaks attributed to Ag element, while the other weak peaks that appeared in all samples was attributed to $\mathrm{C}, \mathrm{O}$ and $\mathrm{N}$ it may be due to the glass slide and PDA layer. The results were presented in a table attached with each analysis. The Ag element appears in all samples before and after Ag NW coating with PDA layer and after it has been exposed to 20 explosions, indicating that technique of decorating Ag NPs on the AgNW@PDA surface was successful. The content of the detected Ag element decreased clearly from $96.74 \%$ to $74.34 \%$ due to that Ag NWs have been coated with PDA layer. Then increased to 79.69 after it has been exposed to 20 explosions. On the other hand, the content of the PDA layer decreases with the occurrence of an explosion that decorates the surface of the AgNW@PDA with Ag NPs. This result corresponds to TEM images in Fig. 4, which demonstrate the formation of AgNW@PDA@AgNPs nanostructures.

\section{STEM image analyses}

Fig. 4 demonstrates the particle size and structure information morphology of the Ag NPs and nanostructures AgNw@PDA and AgNw@PDA@ AgNPs. As shows in Fig. 4(a), an average size of Ag NPs was $40 \mathrm{~nm}$. The Ag NPs were nearly spherical and slightly agglomerated. As clearly seen from Fig. 4(b), the PDA layer could be fixed onto Ag NWs surface and the Ag NWs have been coated completely to form the AgNW@PDA nanostructure, where the Ag NWs act as a core and PDA formed as an amorphous shell. Fig. 4(b), demonstrates that the PDA molecules are coated
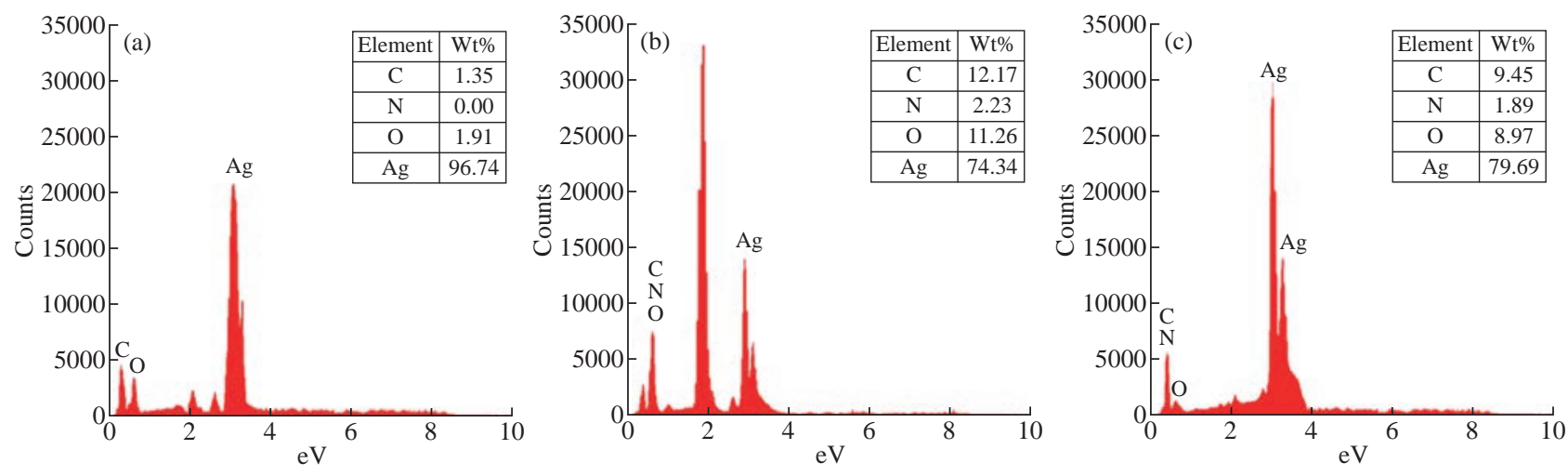

Fig. 3 The EDX spectrum of (a) Ag NW, (b) AgNW@PDA and (c) AgNW@PDA@AgNPs. 

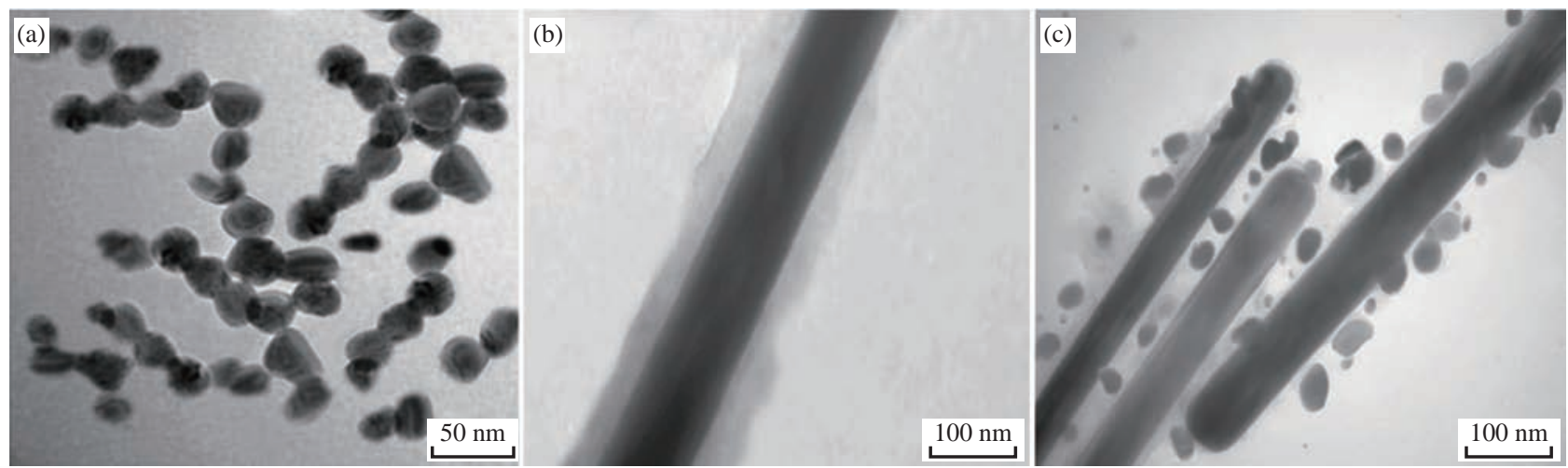

Fig.4 STEM images of (a) Ag NPs, (b) AgNW@PDA and (c) AgNW@PDA@AgNPs.

on the surface of Ag NWs, and creation of assembled functional PDA layer by self-polymerization. It is interesting to mention that the thickness of PDA layer can be controlled simply by changing the reaction time, so that thickness increases as the reaction time increases [37]. On the STEM scale in Fig. 4(b), we can estimate that the PDA layer thickness is $\sim 50 \mathrm{~nm}$. Fig. 4(c) shows that the density number and the distribution of Ag NPs that's decorated on AgNW@PDA surface were randomly. The Ag NPs were adhered to the surface of AgNW@PDA very tightly to form the nanostructures AgNW@PDA@AgNPs. The nanogaps among nanoparticles on the surface were not uniform in distance. The PDA layer served as a spacer molecule (nanogap) to separate the two metals Ag NWs and Ag NPs. The density number of the Ag NPs on the surface of the AgNW@PDA nanostructures was depended on number of explosions.

\section{FESEM image analyses}

Fig. 5(a) illustrates that the Ag NWs have a uniform straight morphology and smooth surface. In Fig. 5(b), the AgNW@AgNPs nanostructures displayed a relatively irregular morphology, which indicated that Ag NWs surface was decorated by Ag
NPs. After performing the EEW technique, many Ag NPs appeared on the surface of Ag NWs along the long axis, and all of the Ag NWs have successfully been decorated with Ag NPs and formed a series of AgNW@AgNPs nanostructures. Also, can be seen that the scattered Ag NPs were adhered tightly to the Ag NWs surface randomly and formed irregular rough surface contain plentiful Ag NPs.

\section{UV-visible absorption spectra}

Fig. 6 illustrates the UV-visible absorption spectra of Ag NPs with different concentrations of 2 $\times 10^{-5}, 4 \times 10^{-5}, 6 \times 10^{-5}, 8 \times 10^{-5}, 1 \times 10^{-4} \mathrm{M}$ with and without R6G dye $\left(1 \times 10^{-6} \mathrm{M}\right)$. The absorption spectra of prepared nanoparticles in deionized water confirm the position of absorption due to surface plasmon resonance (SPR) [38]. SPR peaks were distinctive and their locations are almost constant. In Fig. 6(a), the surface Plasmon resonance of the Ag NPs appeared at $404 \mathrm{~nm}$, and the SPR peak intensity was increased with increasing of Ag NPs concentrations. The increase in the intensity of SPR peaks is due to the increase in resonance between the collective oscillations of conduction electrons with incident electromagnetic field [39]. On the other hand, as shows in Fig. 6(b),
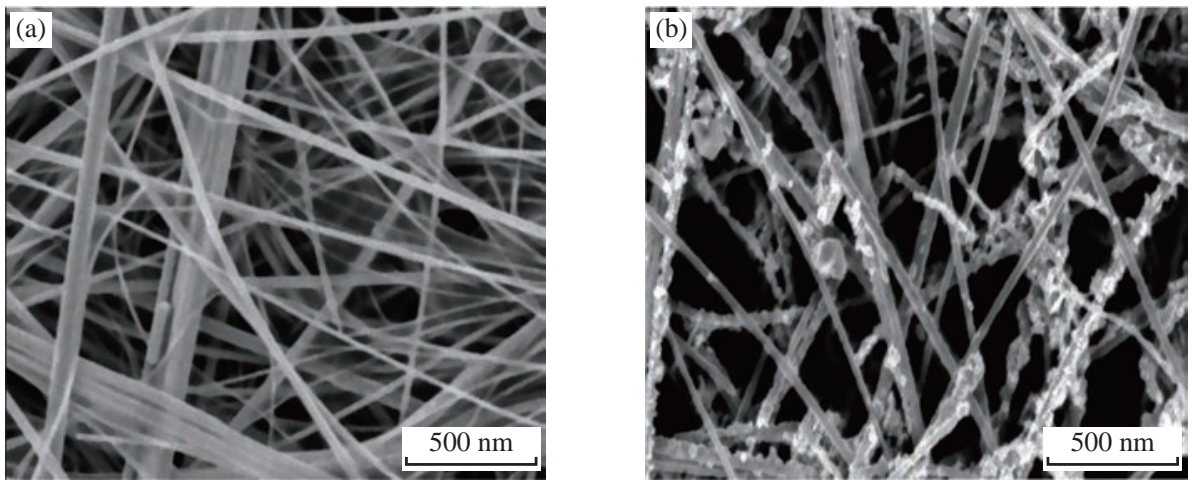

Fig. 5 FESEM images of (a) Ag NWs and (b) AgNW@AgNPs. 

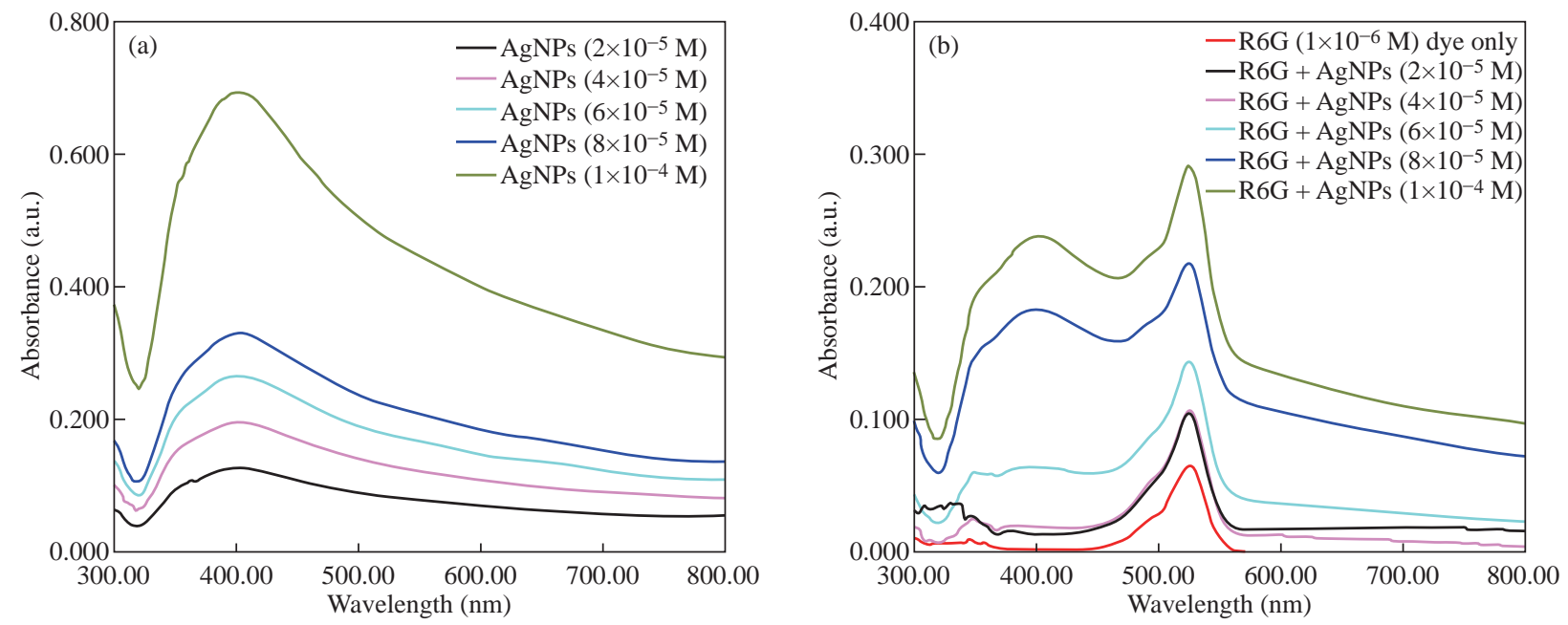

Fig. 6 The UV-visible absorption spectra of different Ag NPs concentrations (a) without R6G dye and (b) with R6G dye $\left(1 \times 10^{-6} \mathrm{M}\right)$.

the absorption peak position of R6G $\left(1 \times 10^{-6} \mathrm{M}\right)$ was at $525 \mathrm{~nm}$. The absorption peak of the R6G dye was increased with increasing of Ag NPs concentration. The SPR peaks of the Ag NPs were decreased after Ag NPs had mixed with the dye because the energy is transferred from the nanoparticles to the dye [40].

Fig. 7 illustrates the UV-visible absorption spectra of Ag NWs at different concentrations of 1 $\times 10^{-4}, 2 \times 10^{-4}, 3 \times 10^{-4}, 4 \times 10^{-4}$ and $5 \times 10^{-4} \mathrm{M}$ with and without R6G dye $\left(1 \times 10^{-6} \mathrm{M}\right)$. In Fig. 7(a), the SPR of the Ag NWs has two peaks, the first appeared in $351 \mathrm{~nm}$, due to the longitudinal Plasmon resonance absorption of the Ag NWs and the second at $374 \mathrm{~nm}$ due to transverse Plasmon resonance of Ag NWs [41]. The intensity of these peaks increased with the increasing of the Ag NWs concentration. For other hand, as shows in Fig. 7(b), the R6G dye molecules have absorption peaks at $524 \mathrm{~nm}$ and the intensity of this peak increased with the increasing of the Ag NWs concentration. This behaviour can be illustrated as follows. Plasmonic metal nanostructures have shown to be able to enhance optical processes. The enhanced effect of surface absorption is enhanced based on the excitation of localized surface plasmons giving enhanced electromagnetic fields; thus, the molecules placed inside this enhanced field adsorbed on the nanostructures will be excited more often due to the electromagnetic field enhancement of the incident light on the active substrate, which leads to improve the absorption [40].

Fig. 8 illustrates that two observed distinctive SPR peaks appeared at about $351 \mathrm{~nm}$ and $373 \mathrm{~nm}$ in the absorption spectrum of Ag NWs. The peaks nearly vanished when the Ag NWs were coated with PDA layer by self-polymerization as seen clearly in the absorption spectrum of AgNW@PDA in Fig.
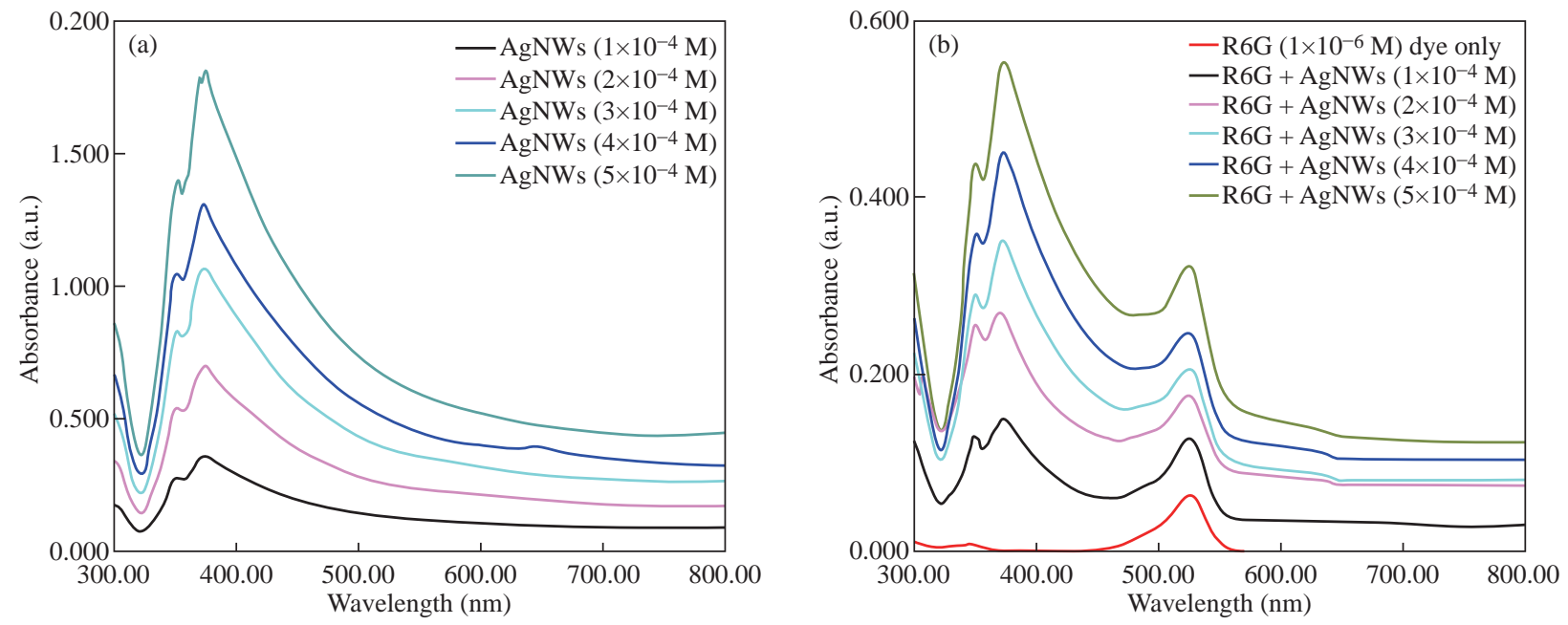

Fig. 7 The UV-visible absorption spectra of different Ag NWs concentrations (a) without R6G dye and (b) with R6G dye $\left(1 \times 10^{-6} \mathrm{M}\right)$. 

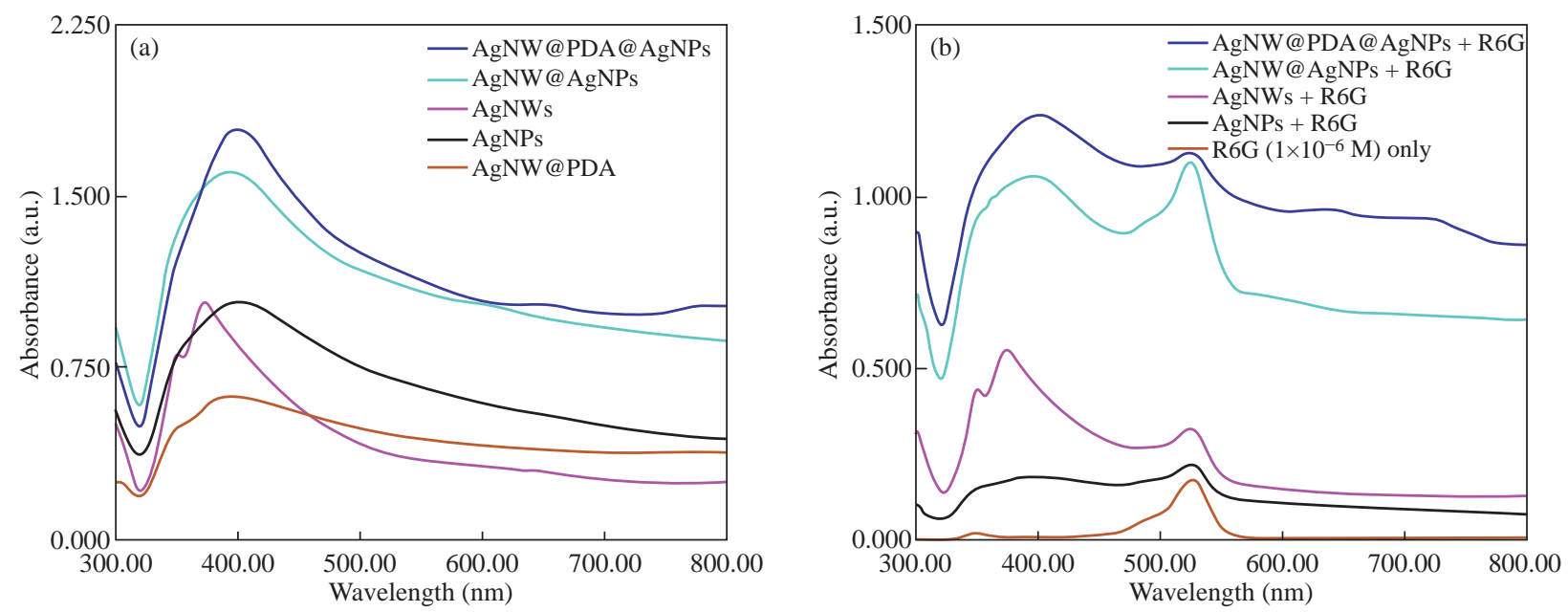

Fig. 8 The UV-visible absorption spectrum of R6G dye and R6G with Ag NPs, Ag NWs, AgNW@AgNPs and AgNW@PDA@ AgNPs nanostructures.

8(a). The absorption peaks of AgNW@AgNPs and AgNW@PDA@AgNPswere clearly observed at 391 and $400 \mathrm{~nm}$ respectively. They have wider and higher absorption peaks than the rest, indicating of the plentiful Ag NPs formation on the surfaces of the Ag NWs and the PDA layer. The stimulation to the enhancement of the electromagnetic field arises due to the coupling between the localized surface plasmon (LSP) of Ag NWs and the dense hot spots of the AgNW@AgNPs that formed by the decoration of Ag NPs on the surface of Ag NWs. While the nanostructures were constructed by Ag NPs decorated AgNW@PDA to formAgNW@PDA@AgNPs nanostructures that have nanogaps between Ag NWs and Ag NPs that have surface plasmon polariton (SPP). The electromagnetic field was enhanced remarkably due to the coupling of LSP-LSP and LSP-SPP [42]. Moreover, the closely adjacent Ag NPs usually exhibited collective LSP mode whose electromagnetic field was much stronger than that of non-adjacent $\mathrm{Ag}$ NPs; therefore,AgNW@AgNPs and AgNW@PDA@ AgNPs nanostructures can absorb more energy in the UV-visible range. The intensity of the electromagnetic field increased with decreasing of the distance between nanoparticles. This phenomenon needs more research.

Two peaks of the Ag NWs at 351 and $373 \mathrm{~nm}$ became less obvious due to the overlap of the two absorption peaks with the absorption peak of the Ag NPs after forming nanostructures. An absorption peak of nanostructures became wider when nanostructures mixed with the R6G dye. As clearly seen, in the Fig. 8(b), the R6G dye molecules have absorption peaks at $524 \mathrm{~nm}$ and the intensity of this peak increased when R6G dye mixed with Ag NPs, Ag NWs,
AgNW@AgNPs and AgNW@PDA@AgNPs, where the intensities were $0.237,0.298,1.1$ and 1.128 respectively.

The SPR peaks of the AgNW@PDA@AgNPs nanostructures were enhanced more than other nanostructures due to the SPR of metal nanostructures was greatly sensitive to the dielectric environment around surface morphology [36].

\section{Fluorescence emission spectra}

As mentioned previously, the efficiency of energy transfer depends on the spectral overlap of the absorption spectra of the acceptor (Ag NPs, Ag NWs,AgNW@AgNPs and AgNW@PDA@AgNPs nanostructures) and the photoluminescence emission spectrum of the emitter (R6G dye). Fig. 9 shows the partial spectral overlap between the absorption

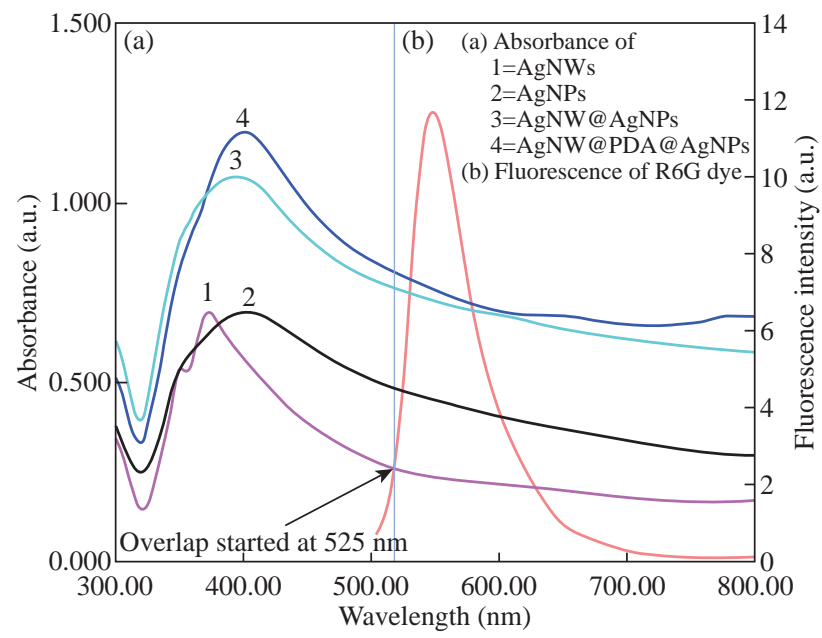

Fig. 9 The spectral comparison between (a) absorption spectra of Ag NPs, Ag NWs, AgNW@AgNPs and AgNW@PDA@ AgNPs and (b) fluorescence spectrum of R6G. 
spectra of the acceptor (Ag NPs, Ag NWs, AgNW@ AgNPs and AgNW@PDA@AgNPs nanostructures and emission spectrum of R6G dye. An absorption spectrum of Ag NPs, Ag NWs, AgNW@AgNPs and AgNW@PDA@AgNPs nanostructures exhibit reasonable overlap with the PL spectrum of the R6G dye (started from $525 \mathrm{~nm}$ to $540 \mathrm{~nm}$ ). So, the wavelength of excitation of fluorescence samples was $532 \mathrm{~nm})$.

In order to understand the energy transfer efficiency from R6G dye molecules to the nanostructures, stable concentration $\left(1 \times 10^{-6} \mathrm{M}\right)$ of the dye has been taken and mixed with nanostructures. Fluorescence spectra of R6G dye that mixed with nanostructures are measured using a spectrofluorophotometer (Shimadzu RF-5301 PC) at an excitation wavelength $532 \mathrm{~nm}$. Fig. 10(a) shows the fluorescence spectra of R6G dye mixed with different Ag NPs concentrations of $2 \times 10^{-5}, 4 \times 10^{-5}$, $6 \times 10^{-5}, 8 \times 10^{-5}$ and $1 \times 10^{-4}$ M. Fig. 10(b) shows the fluorescence spectra of R6G dye mixed with different Ag NWs concentrations of $1 \times 10^{-4}, 2 \times 10^{-4}, 3$ $\times 10^{-4}, 4 \times 10^{-4}$ and $5 \times 10^{-4} \mathrm{M}$. It is clear from Fig. 10(a) and (b) that the fluorescence intensity of R6G dye has been quenched obviously with an increase in the concentration of both Ag NPs and Ag NWs in the R6G dye solution, and the quenching increases as the concentration of both Ag NPs and Ag NWs increases in the mixture.

Fig. 11(a) shows the fluorescence spectra of R6G dye mixed with nanostructures AgNW@ AgNPs. The increase in the number of explosions represents an increase in the density number of Ag NPs on the surface of the Ag NW. Fig. 11(b) shows the fluorescence spectra of R6G dye mixed with
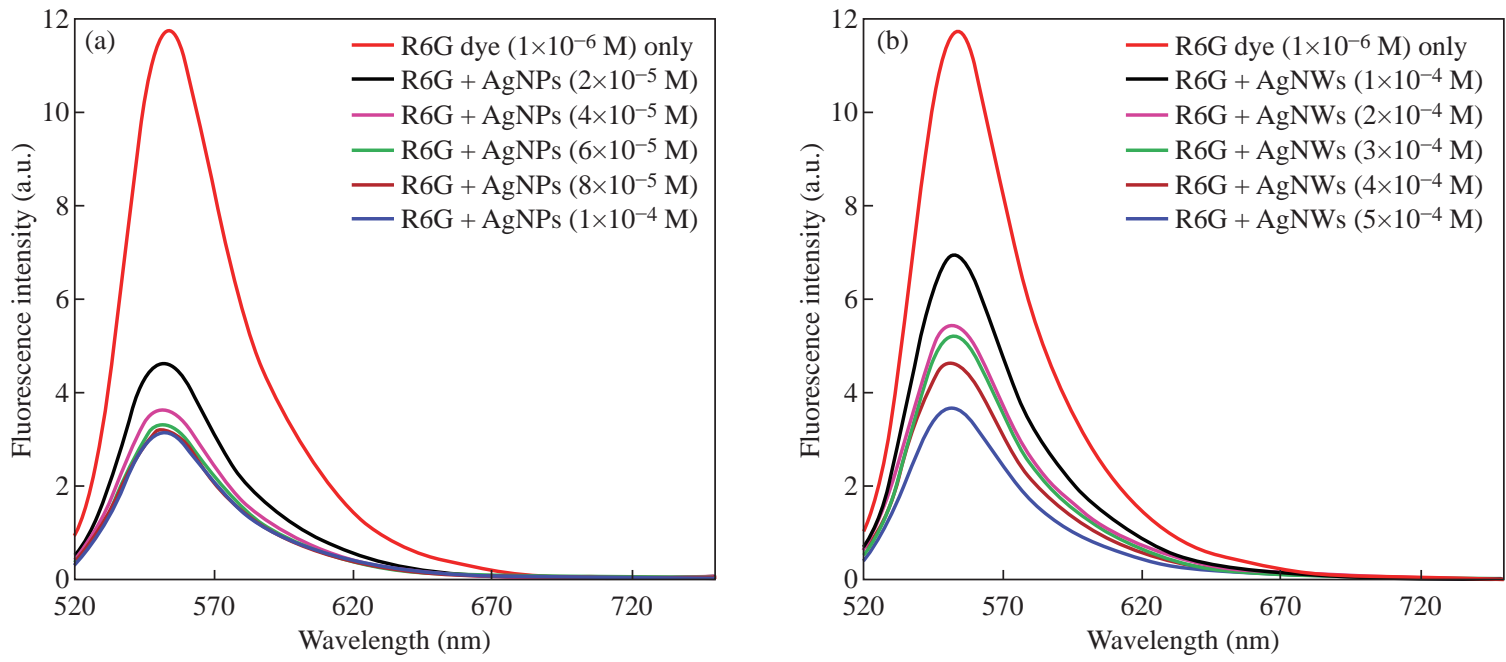

Fig. 10 Fluorescence spectra of R6G dye solution $\left(1 \times 10^{-6} \mathrm{M}\right)$ quenched after mixed with (a) Ag NPs with different concentrations and (b) Ag NWs with different concentrations.
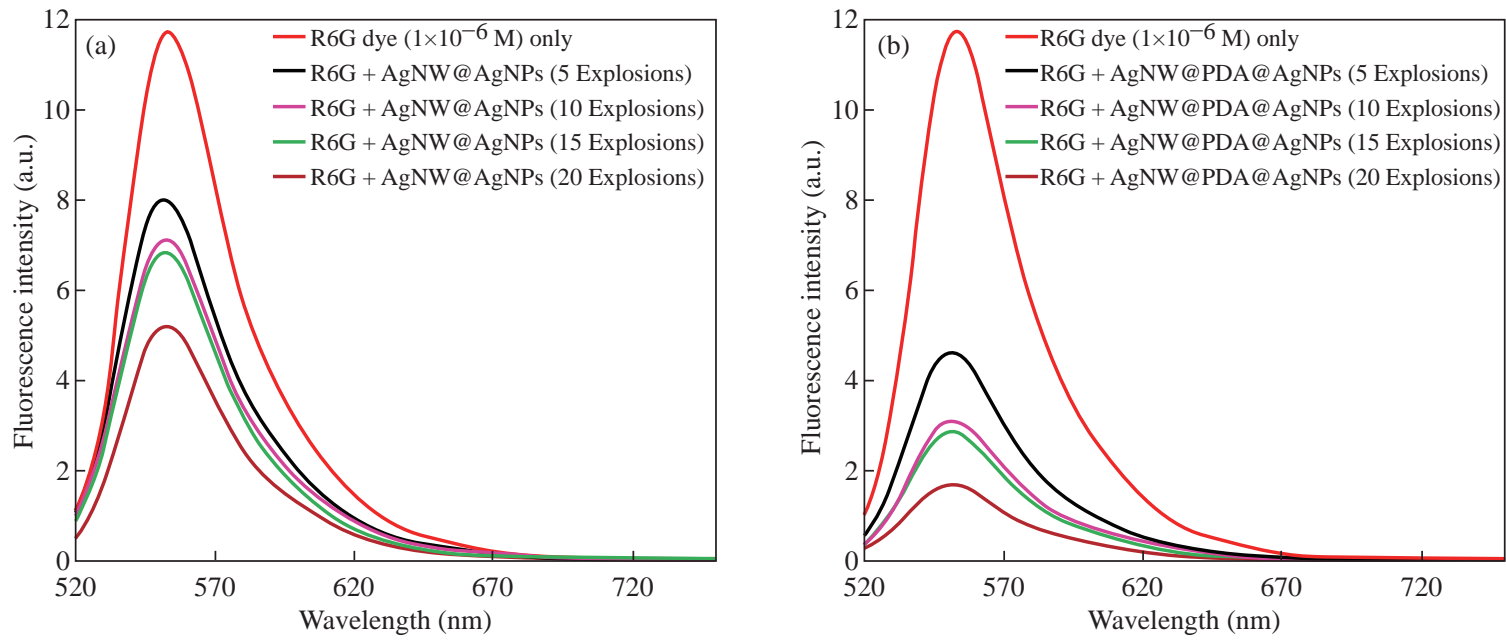

Fig. 11 Fluorescence spectra of R6G dye solution $\left(1 \times 10^{-6} \mathrm{M}\right)$ quenched after mixed with nanostructures of (a) AgNW@AgNPs and (b)AgNW@PDA@AgNPs. 
nanostructures AgNW@PDA@AgNPs. It appears from Fig. 11(a) and (b) that the fluorescence intensity of R6G dye has been quenched obviously as the number of explosions increased.

The quantitative relationship between the concentration of the acceptor (Ag NPs or Ag NWs) and intensity of the donor fluorescence (Rh6G dye) can be acquired from the well-known Stern-Volmer equation:

$\mathrm{F}_{\mathrm{D}} / \mathrm{F}_{\mathrm{DA}}=1+\mathrm{K}_{\mathrm{SV}}[\mathrm{A}]=1+\mathrm{K}_{\mathrm{q}} \tau_{0}[\mathrm{~A}]$,

where $F_{D}$ and $F_{D A}$ are the relative fluorescence intensity of the donor (R6G dye) in the absence and presence of the acceptor (Ag NPs or Ag NWs) respectively, $\mathrm{K}_{\mathrm{SV}}$ is the Stern-Volmer dynamic-quenching constant, $\tau_{0}$ is the lifetime of the excited donor (R6G dye) in the absence of the acceptor, [A] is the acceptor concentration (Ag NPs or Ag NWs).

All the results depicted in Fig. 12 have been subjected to the well-known Stern-Volmer equation. The values of quenching constants in the R6Gnanostructures system have been calculated for four states. For R6G + Ag NPs system, the ratio fluorescence intensity of the donor alone (R6G dye) at concentration of $1 \times 10^{-6} \mathrm{M}$ to the fluorescence intensity

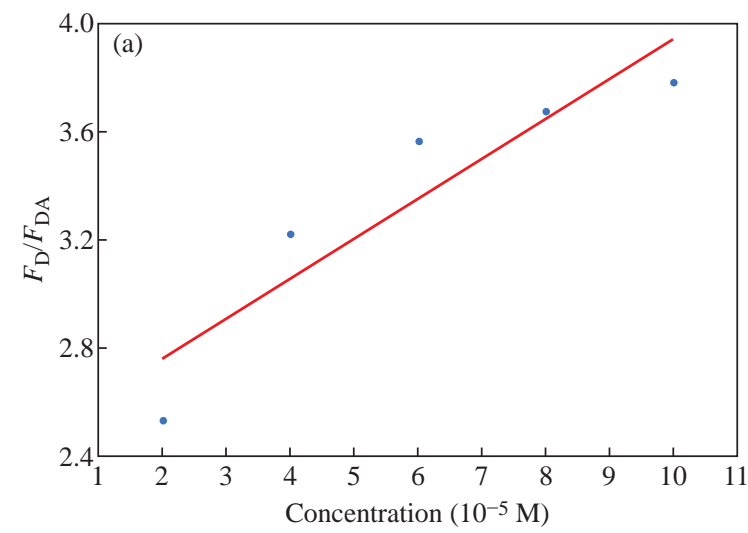

of the mixture (R6G + Ag NPs) with acceptor (Ag NPs) concentrations of $2 \times 10^{-5}, 4 \times 10^{-5}, 6 \times 10^{-5}, 8 \times 10^{-5}$ and $1 \times 10^{-4} \mathrm{M}$ as in Fig. 12(a), where the quenching constant of this system was $\mathrm{K}_{\mathrm{sv}}=1.476 \times 10^{4} \mathrm{M}^{-1}$. For $\mathrm{R} 6 \mathrm{G}+\mathrm{Ag}$ NWs system, the ratio fluorescence intensity of the donor alone (R6G dye) at concentration of $1 \times$ $10^{-6} \mathrm{M}$ to the fluorescence intensity of the mixture (R6G + Ag NWs) with acceptor (Ag NWs) concentrations of $1 \times 10^{-4}, 2 \times 10^{-4}, 3 \times 10^{-4}, 4 \times 10^{-4}$ and $5 \times 10^{-4} \mathrm{M}$ as in Fig. 12(b), where the quenching constant of this system was $\mathrm{K}_{\mathrm{sv}}=0.342 \times 10^{4} \mathrm{M}^{-1}$. The nearly linear conduct of Fig. 12 denotes that there is no hyper-quenching.

For R6G + AgNW@AgNPs system, the ratio fluorescence intensity of the donor alone (R6G dye) at concentration of $1 \times 10^{-6} \mathrm{M}$ to the fluorescence intensity of the mixture (R6G + AgNW@AgNPs) with increasing of number of explosions (5, 10, 15 and 20 explosions) as in Fig. 13(a), where the quenching constant is a function of the number of explosions. For a R6G+AgNW@PDA@AgNPs system in Fig. 13(b), the same procedure is used for system R6G+AgNW@ AgNPs, where the quenching constant is a function of the number of explosions.

The energy transfer efficiencies from R6G dye to Ag

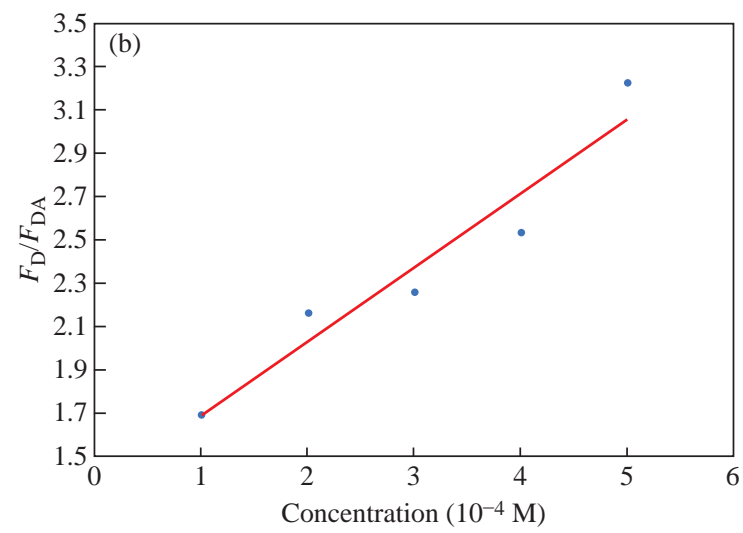

Fig. 12 Stern-Volmer plot for (a) R6G + Ag NPs system and (b) R6G + Ag NWs system.
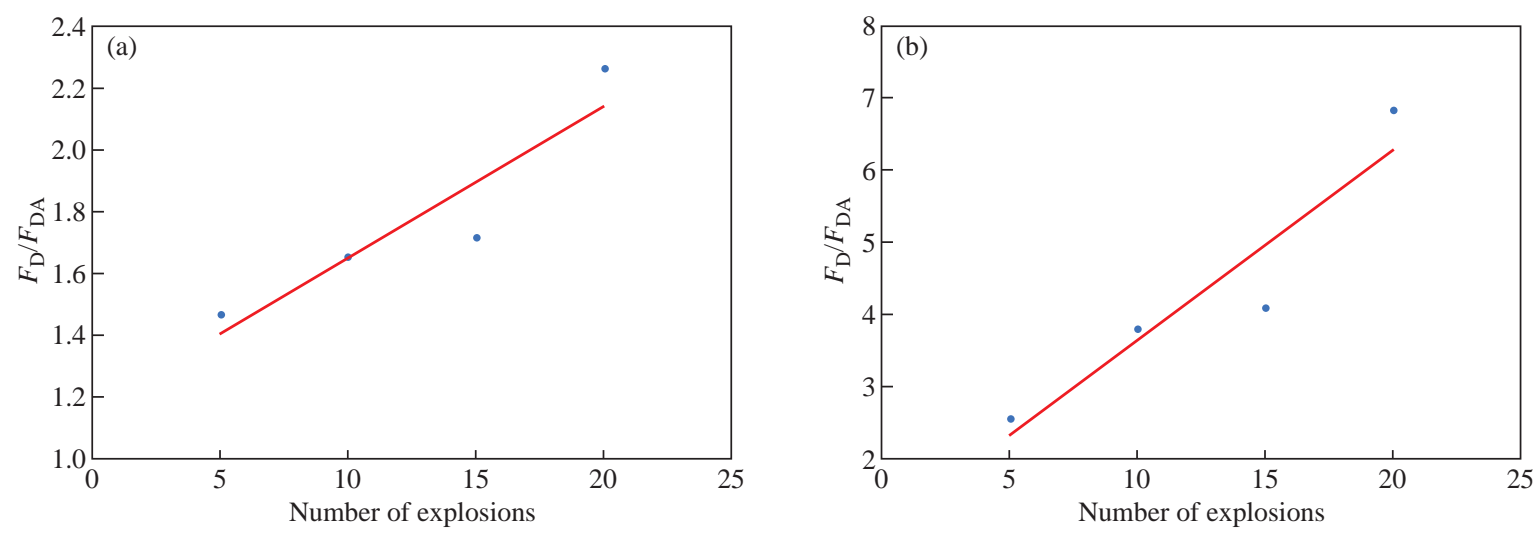

Fig. 13 Stern-Volmer plot for (a) R6G +AgNW@AgNPs system and (b) R6G + AgNW@PDA@AgNPs system. 
NPs, Ag NWs, AgNW@AgNPs or AgNW@PDA@ AgNPs are calculated from the fluorescence measured can be evaluated using the equation:

$\phi_{\mathrm{ET}}=1-\mathrm{F}_{\mathrm{DA}} / \mathrm{F}_{\mathrm{D}}$.

The energy transfer efficiencies obtained and the corresponding acceptor concentrations are listed in Table 1.

The energy transfer efficiencies obtained and the corresponding acceptor concentrations (number of explosions) are listed in Table 2.

From the above results it is clear to us that the energy transfer efficiency increases as the concentration and number of Ag NPs increases in all systems. The energy transfer efficiency is good in R6G + Ag NPs system and very good in R6G+AgNW@PDA@ AgNPs system. Fluorescence quenching efficiency is related to the size and number density number of Ag NPs [43]. This is due to the phenomenon of selfassembly of R6G molecules on Ag NPs [44]. The selfassembly of R6G molecules on Ag NPs increases as concentration of Ag NPs increases. Also, Ag NPs on the surface of the PDA layer achieves the phenomenon of self-assembly of R6G molecules on Ag NPs and
PDA layer. Thus, the nanostructures AgNW@PDA@ AgNPs with a high-density number of Ag NPs have the best fluorescence quenching.

The distance in nano unit (R) between the R6G molecules and nanomaterial for different concentrations of Ag NPs and Ag NWs can be calculated using the equation $[45,46]$ :

$\mathrm{R}_{0}(\mathrm{~nm})=0.735 /[\mathrm{A}]^{1 / 3}$.

The values of the different concentrations of Ag NPs and Ag NWs in the R6G + Ag NWs system and R6G + Ag NWs system and the corresponding dyenanomaterial distances are given in Table 3.

The distance $\mathrm{R}$ decreases as the concentration of nanomaterial increases. So, the energy transfer efficiency increases as the distance decreases. The Ag NPs prepared by EEW technique and the nanostructure of PDA coated Ag NWs and decorated with Ag NPs by EEW technique have achieved the best energy transfer efficiency. Our results indicate that our systems can sense a distance around the metal nanoparticles $(\approx 27.2$ $\mathrm{nm})$, and thus the nanoparticles-based surface energy transfer (NSET) mechanism is dominated rather than Förster resonance energy transfer (FRET) mechanism.

Table 1 The energy transfer efficiencies of R6G + Ag NPs system and R6G + Ag NWs system

\begin{tabular}{|c|c|c|c|c|c|}
\hline \multicolumn{3}{|c|}{ R6G + Ag NPs system } & \multicolumn{3}{|c|}{ R6G + Ag NWs system } \\
\hline Concentration & $\mathrm{F}_{\mathrm{DA}} / \mathrm{F}_{\mathrm{D}}$ & $\phi_{\mathrm{ET}}$ & Concentration & $\mathrm{F}_{\mathrm{DA}} / \mathrm{F}_{\mathrm{D}}$ & $\phi_{\mathrm{ET}}$ \\
\hline $2 \times 10^{-5} \mathrm{M}$ & 0.40 & $60 \%$ & $1 \times 10^{-4} \mathrm{M}$ & 0.58 & $42 \%$ \\
\hline $4 \times 10^{-5} \mathrm{M}$ & 0.31 & $69 \%$ & $2 \times 10^{-4} \mathrm{M}$ & 0.46 & $54 \%$ \\
\hline $6 \times 10^{-5} \mathrm{M}$ & 0.28 & $72 \%$ & $3 \times 10^{-4} \mathrm{M}$ & 0.44 & $56 \%$ \\
\hline $8 \times 10^{-5} \mathrm{M}$ & 0.27 & $73 \%$ & $4 \times 10^{-4} \mathrm{M}$ & 0.40 & $60 \%$ \\
\hline $1 \times 10^{-4} \mathrm{M}$ & 0.26 & $74 \%$ & $5 \times 10^{-4} \mathrm{M}$ & 0.31 & $69 \%$ \\
\hline
\end{tabular}

Table 2 The energy transfer efficiencies of R6G+AgNW@AgNPs system and R6G+AgNW@PDA@AgNPs system

\begin{tabular}{|c|c|c|c|c|c|}
\hline \multicolumn{3}{|c|}{ R6G + AgNW@AgNPs system } & \multicolumn{3}{|c|}{ R6G + AgNW@PDA@AgNPs system } \\
\hline Number of explosions & $\mathrm{F}_{\mathrm{DA}} / \mathrm{F}_{\mathrm{D}}$ & $\phi_{\mathrm{ET}}$ & Number of explosions & $\mathrm{F}_{\mathrm{DA}} / \mathrm{F}_{\mathrm{D}}$ & $\phi_{\mathrm{ET}}$ \\
\hline 5 & 0.68 & $32 \%$ & 5 & 0.40 & $60 \%$ \\
\hline 10 & 0.61 & $39 \%$ & 10 & 0.27 & $73 \%$ \\
\hline 15 & 0.58 & $42 \%$ & 15 & 0.25 & $75 \%$ \\
\hline 20 & 0.44 & $56 \%$ & 20 & 0.15 & $85 \%$ \\
\hline
\end{tabular}

Table 3 The distance R between the R6G molecules and nanomaterials of the R6G+AgNPs system and R6G+Ag NWs system

\begin{tabular}{|c|c|c|c|c|c|}
\hline \multicolumn{3}{|c|}{ R6G + Ag NPs system } & \multicolumn{3}{|c|}{ R6G + Ag NWs system } \\
\hline$[\mathrm{A}]$ & {$[\mathrm{A}]^{1 / 3}$} & $\mathrm{R}_{0}(\mathrm{~nm})$ & [A] & {$[\mathrm{A}]^{1 / 3}$} & $\mathrm{R}_{0}(\mathrm{~nm})$ \\
\hline $2 \times 10^{-5} \mathrm{M}$ & 0.027 & 27.2 & $1 \times 10^{-4} \mathrm{M}$ & 0.046 & 16.0 \\
\hline $4 \times 10^{-5} \mathrm{M}$ & 0.034 & 21.6 & $2 \times 10^{-4} \mathrm{M}$ & 0.058 & 12.7 \\
\hline $6 \times 10^{-5} \mathrm{M}$ & 0.039 & 18.8 & $3 \times 10^{-4} \mathrm{M}$ & 0.067 & 11.0 \\
\hline $8 \times 10^{-5} \mathrm{M}$ & 0.043 & 17.1 & $4 \times 10^{-4} \mathrm{M}$ & 0.074 & 9.9 \\
\hline $1 \times 10^{-4} \mathrm{M}$ & 0.046 & 16.0 & $5 \times 10^{-4} \mathrm{M}$ & 0.079 & 9.3 \\
\hline
\end{tabular}


This process is affected by concentration increasing of Ag NPs and coated morphology of Ag NWs by PDA layer that decorated by Ag NPs. A better and indepth study of this phenomenon would aid research advances and applications of this system for imaging and sensing.

\section{Conclusions}

It can be concluded that EEW technique is a simple and efficient technique to fabricate nanostructures. The fabricated nanostructures can be used to test the fluorescence spectra. The experimental results showed that the fluorescence intensity quenched with increasing concentration and density number of Ag NPs. The distance between the dye molecules and the nanostructures decreased as the concentration and density number of Ag NPs increased in the mixture. The energy transfer efficiency of nanostructures was obtained, where the nanostructure AgNW@PDA@ AgNPs achieved the best energy transfer efficiency of $85 \%$. Our results indicate that this nanostructure can sense a distance around the metal nanoparticles $(\approx 27.2 \mathrm{~nm}$ ), and thus the nanoparticles-based surface energy transfer (NSET) mechanism is dominating rather than Förster resonance energy transfer (FRET) mechanism. This process is affected by concentration increasing of Ag NPs and coated morphology of Ag NWs by PDA layer decorated by Ag NPs. The findings can be utilized in the large field of bio diagnostics and biochemistry. Regardless of bio-applications, the quenching mechanisms and rates are also of interest for SERS, (dye-sensitized) solar cells or nanooptics. However, we see the best potential in bio-sensing by managing the quenching rate by adjusting the shape or the concentration of nanostructures.

\section{References}

[1] L. Kumar, R. Medwal, P. Sen, et al., Processing temperature driven morphological evolution of $\mathrm{ZnO}$ nanostructures prepared by electro-exploding wire technique. Materials Research Express, 2014, 1(1): 015045.

[2] A.S. Wasfi, H.R. Humud, and N.K. Fadhil, Synthesis of core-shell Fe3O4-Au nanoparticles by electrical exploding wire technique combined with laser pulse shooting. Optics and Laser Technology, 2019, 111: 720-726.

[3] H. Deng, H. Yu, Distance-dependent fluorescence quenching on a silver nanoparticle surface. Chem. Lett, 2019, 48: 1504-1506.

[4] S.F. Haddawi, H.R. Humud, and S.M. Hamidi, Signature of plasmonic nanoparticles in multi-wavelength low power random lasing. Optics and Laser Technology, 2019,
121: 105770.

[5] X. Han, K. Xu, O. Taratula, et al., Applications of nanoparticles in biomedical imaging. Nanoscale, 2019, 11: 799-819.

[6] I. Khan, K. Saeed, and I. Khan, Nanoparticles: Properties, applications and toxicities. Arabian Journal of Chemistry, 2019, 12(7): 908-931.

[7] M. Azharuddin, G.H. Zhu, D. Das, et al., A repertoire of biomedical applications of noble metal nanoparticles. Chem. Commun, 2019, 55: 6964-6996.

[8] C. Sönnichsen, B.M. Reinhard, J. Liphardt, et al., A molecular ruler based on plasmon coupling of single gold and silver nanoparticles. Nat. Biotechnol, 2005, 23: 741745.

[9] B. Le Ouay, F. Stellacci, Antibacterial activity of silver nanoparticles: a surface science insight. Nano Today, 2015, 10: 339-354.

[10] D. McShan, P.C. Ray, and H. Yu, Molecular toxicity mechanism of nanosilver. J. Food Drug Anal, 2014, 22: 116-127.

[11] P. AshaRani, G. Low Kah Mun, M.P. Hande, et al., Cytotoxicity and genotoxicity of silver nanoparticles in human cells. ACS Nano, 2008, 3: 279-290.

[12] G.A. Sotiriou, S.E. Pratsinis, Antibacterial activity of nanosilver ions and particles. Environ. Sci. Technol, 2010, 44: 5649-5654.

[13] M. Rai, A. Yadav, and A. Gade, Silver nanoparticles as a new generation of antimicrobials. Biotechnol. Adv, 2009, 27: 76-83.

[14] T.R. Jensen, M.D. Malinsky, C.L. Haynes, et al., Nanosphere lithography: tunable localized surface plasmon resonance spectra of silver nanoparticles. $J$. Phys. Chem. B, 2000, 104: 10549-10556.

[15] D. Graham, D.G. Thompson, W.E. Smith, et al., Control of enhanced Raman scattering using a DNA-based assembly process of dye-coded nanoparticles. Nat. Nanotechnol, 2008, 3: 548-551.

[16] T. Miclaus, V.E. Bochenkov, R. Ogaki, et al., Spatial mapping and quantification of soft and hard protein coronas at silver nanocubes. Nano Lett, 2014, 14: 20862093.

[17] C.D. Walkey, J.B. Olsen, F. Song, et al., Protein corona fingerprinting predicts the cellular interaction of gold and silver nanoparticles. ACS Nano, 2014, 8: 2439-2455.

[18] Z. Zhong, S. Patskovskyy, P. Bouvrette, et al., The surface chemistry of Au colloids and their interactions with functional amino acids. J. Phys. Chem. B, 2004, 108: 4046-4052.

[19] C.D. Walkey, J.B. Olsen, H. Guo, et al., Nanoparticle size and surface chemistry determine serum protein adsorption and macrophage uptake. J. Am. Chem. Soc, 2012, 134: 2139-2147.

[20] X. Huang, J. Song, B.C. Yung, et al., Ratiometric optical nanoprobes enable accurate molecular detection and imaging. Chem. Soc. Rev, 2018, 47: 2873-2920.

[21] W. Wu, G.C. Bazan and Bin, Conjugated-polymeramplified sensing, imaging, and therapy. Chem, 2017, 2(6): 760-790.

[22] S.A. Khan, A.M. Asiria, and S.H. Al-Thaqafya, Optical properties and fluorescence quenching of biologically active ethyl 4-(4-N,N-dimethylamino phenyl)-2-methyl-5oxo-4,5-dihydro-1H-indeno[1,2-b]pyridine-3-carboxylate (DDPC) dye as a probe to determine CMC of surfactants. RSC Adv, 2016, 6: 102218-102225.

[23] T.L. Jennings, M.P. Singh, and G.F. Strouse, Fluorescent lifetime quenching near $\mathrm{D}=1.5 \mathrm{~nm}$ gold nanoparticles: Probing NSET validity. J Am Chem Soc, 2006, 128(16): 5462-5467.

[24] Y. Chen, M.B. O’Donoghue, Y.F. Huang, et al., A surface energy transfer nanoruler for measuring binding site 
distances on live cell surfaces. J. Am.Chem. Soc, 2010, 132: 16559.

[25] S. Batabyal, T. Mondol, K. Das, et al., Förster resonance energy transfer in a nanoscopic system on a dielectric interface. Nanotechnology, 2012, 23(49): 495402.

[26] B.N.J. Persson, N.D. Lang, Electron-hole-pair quenching of excited states near a metal. Phys. Rev. B, 1982, 26: 5409.

[27] S. Liu, D. Leech, and H. Ju, Application of colloidal gold in protein immobilization, electron transfer, and biosensing. Anal. Lett, 2003, 36(1).

[28] C.S. Yun, A. Javier, T. Jennings, et al., Nanometal surface energy transfer in optical rulers, breaking the FRET barrier. J. Am. Chem. Soc, 2005, 127(9): 3115-3119.

[29] T. Sen, A. Patra, Recent advances in energy transfer processes in gold-nanoparticle-based assemblies. J. Phys. Chem. C, 2012, 116(33): 17307-17317.

[30] V.N. Pustovit, T.V. Shahbazyan, Fluorescence quenching near small metal nanoparticles. J. Chem. Phys, 2012, 136(20): 204701.

[31] H. Yu, Y. Peng, Y. Yang, et al., Plasmon-enhanced lightmatter interactions and applications. npj Comput Mater, 2019, 5(45).

[32] S. Saini, G. Srinivas, and B. Bagchi, Distance and orientation dependence of excitation energy transfer: from molecular systems to metal nanoparticles. J. Phys. Chem. B, 2009, 113(7): 1817-1832.

[33] D. Singha, D.K. Sahu, and K. Sahu, Coupling of molecular transition with the surface plasmon resonance of silver nanoparticles inside the restricted environment of reverse micelles. ACS Omega, 2017, 2(9): 5494-5503.

[34] M. Swierczewska, S. Lee, and X. Chen, The design and application of fluorophore-gold nanoparticle activatable probes. Phys Chem. Chem. Phys, 2011, 13(21): 99299941.

[35] P. Holzmeister, E. Pibiri, J. Schmied, et al., Quantum yield and excitation rate of single molecules close to metallic nanostructures. Nat Commun, 2014, 5: 5356.

[36] Z. Zhang, T. Si, J. Liu, et al., Controllable synthesis of AgNWs@PDA@AgNPs core-shell nanocobs based on a mussel-inspired polydopamine for highly sensitive SERS detection. RSC Adv, 2018, 8: 27349.

[37] H. Yuan, Y. Wang, T. Li, et al., Highly thermal conductive and electrically insulating polymer composites based on polydopamine-coated copper nanowire. Composites
Science and Technology, 2018, 164: 153-159.

[38] S.F. Haddawi, H.R. Humud, and S.M. Hamidi, Tunable low power piezo-plasmonic random laser under external voltage. Optik - International Journal for Light and Electron Optics, 2020, 207: 164482.

[39] J. Jana, M. Ganguly, and T. Pal, Enlightening surface plasmon resonance effect of metal nanoparticles for practical spectroscopic application. RSC Adv., 2016, 6: 86174-86211.

[40] Barzan, Mohammad, Hajiesmaeilbaigi, et al., Effect of gold nanoparticles on the optical properties of Rhodamine 6G. The European Physical Journal D, 2016, 70(121).

[41] M.B. Gebeyehu, T.F. Chala, S.Y. Chang, et al., Synthesis and highly effective purification of silver nanowires to enhance transmittance at low sheet resistance with simple polyol and scalable selective precipitation method. RSC Adv, 2017, 7: 16139-16148.

[42] Q. Ma, G. Liu, S. Feng, et al., Interaction properties between different modes of localized and propagating surface Plasmons in a dimer nanoparticle array. Optical Engineering, 2018, 57(8): 087108.

[43] Y. Yang, J. Zhao, G. Weng, et al., Fine-tunable fluorescence quenching properties of core-satellite assemblies of gold nanorod-nanosphere: Application in sensitive detection of $\mathrm{Hg}^{2+}$. Spectrochimica Acta Part A: Molecular and Biomolecular Spectroscopy, 2020, 5(228): 117776.

[44] H. Deng, H. Yu, Self-assembly of rhodamine 6G on silver nanoparticles. Chemical Physics Letters, 2018, 692: 75 80.

[45] M. Inokutti, F. Hiryama, Influence of energy transfer by the exchange mechanism on donor luminescence. $J$. Chem. Phys, 1965, 43: 1978.

[46] N. Shemeena Basheer, Thermal lens probing of distant dependent fluorescence quenching of Rhodamine $6 \mathrm{G}$ by silver nanoparticles. Journal of Luminescence, 2013, 137: 225-229.

Copyright $@$ Fouad Hamzah, Hammad Humud. This is an open-access article distributed under the terms of the Creative Commons Attribution License, which permits unrestricted use, distribution, and reproduction in any medium, provided the original author and source are credited. 